\title{
Chinese Herbal Formulation PHY906
}

\author{
National Cancer Institute
}

\section{Source}

National Cancer Institute. Chinese Herbal Formulation PHY906. NCI Thesaurus. Code C91704.

An oral traditional Chinese herbal formulation in powder form containing a spray-dried preparation of an aqueous extract from the four herbs Scutellaria baicalensis, Glycyrrhiza uralensis, Ziziphus jujuba and Paeonia lactiflora, with potential immunomodulating and chemoprotective activities, and which may be used as an adjuvant for chemotherapeutic agents. Although the mechanisms of action remain to be fully elucidated, PHY906 possesses a wide range of pharmacological activities such as the enhancement of oral uptake of pharmacologically active agents, inhibition of CYP3A4, modulation of certain pro-inflammatory cytokines, enhanced migration of macrophages and lymphocytes, and the inhibition of expression of matrix metalloproteinase (MMP), nuclear factor kappaB (NF-kB), cyclooxygenase (COX), nitric oxide synthase (NOS), beta-glucuronidase, the neurokinin 1 receptor (NK1R; tachykinin receptor 1; TACR1; substance-P receptor) and the delta-opioid receptor. PHY906 may also enhance the anti-tumor effect of other anticancer agents, and may protect the gastrointestinal (GI) mucosa against damage and reduce Gl symptoms. 